\title{
Balance Control for an Active Leg Exoskeleton based on Human Balance Strategies
}

\author{
V. Huynh ${ }^{1,2}$, C. Bidard ${ }^{1}$ and C. Chevallereau ${ }^{2}$ \\ ${ }^{1}$ Interactive Robotics Laboratory of CEA-LIST, France, \\ e-mail:vaiyee.huynh@cea.fr, catherine.bidard@cea.fr \\ ${ }^{2}$ Institut de Recherche en Communications et Cybernetique de Nantes, France, \\ e-mail: christine.chevallereau@irccyn.ecnantes.fr
}

\begin{abstract}
This paper presents an open-loop balance control for an active leg exoskeleton based on human balance strategies, and how the machine can balance itself according to perturbations. The control is designed to balance the exoskeleton with a view to assist a well and able operator that leads the movements of the coupled system \{operator+exoskeleton\}. It is inspired by biomechanic works showing that human balance relies on three strategies : the displacement of the center of mass, the contribution of each leg to produce efforts and stepping. We assimilate the exoskeleton to a Linear Inversed Pendulum model to describe its global behavior, and we use its capture point to identify a loss of balance situation possibly caused by the operator and adapt the reaction of the machine. Thanks to capture point's dynamics regarding to the center of mass and the center of pressure, we are able to control the machine and bring it back into a stable situation.
\end{abstract}

Key words: Balance control, capture point, exoskeleton

\section{INTRODUCTION}

Lower leg exoskeletons are more and more developed whether it be for medical applications such as carrying a paraplegic person and rehabilitation, or for industrial applications to enhance operator's skill [1][2][3][4]. In both case, balance control is essential for the coupled system. At present, for medical purposes, the balance of the system is managed by crutches (Rewalk), by the exoskeleton alone (Rex) or by an external structure for rehabilitation (Lokomat). For load carrying exoskeletons (BLEEX, Hv3), devices where the operator is well and able, the lateral balance is assumed by the operator while the exoskeleton carrying its own weight and an additional load. In our works, we will only consider exoskeletons where the machine has to assist the operator in various ways, in particular, in balance recovery.

Balance control is one of the biggest issues of biped robotic or biomechanic works. Balance means that the system does not fall down and the feet are stationed. Therefore, we take inspiration from these researches [5][6][7] to design a balance control for an exoskeleton to react to external perturbations with a smooth action on the sys- 
tem. The objective is to have a balance control for the machine which will imitate human mechanisms in order to support the operator action and reduce his efforts while moving.

\subsection{Human balance strategies}

Balance control for us as humans seems to be simple and easy as we stand, walk and run everyday without falling down. Researchers in biomechanics reveal different strategies we employ to stay stable. Winter [5] studies human balance based commonly on inverted pendulum, in quiet standing (double support) and subjected to low perturbations. He shows the importance of center of pressure (COP) placement in the base of support shaped by the feet - the weighted average point of all the pressures over the contact area feet/ground -, directly linked to center of mass (COM) placement thanks to ankle and hip strategies in order to maintain COM in safe boundaries. He also shows the importance of load/unload mechanism of each leg to correct the postural sway. These two mechanisms involve different muscles and one prevails over the other depending on the posture. Supposing the ground is horizontal, they are summarized in this first equation :

$$
\mathbf{X}_{C O P, \text { global }}=\alpha_{0} \mathbf{X}_{C O P, 0}+\alpha_{1} \mathbf{X}_{C O P, 1}
$$

with $\alpha_{0}=\frac{R_{n, 0}}{R_{n, 0}+R_{n, 1}}, \alpha_{1}=\frac{R_{n, 1}}{R_{n, 0}+R_{n, 1}}$ and $\alpha_{0}+\alpha_{1}=1$, and where $\mathbf{X}_{C O P, \text { global }}=$ $\left[x_{C O P, \text { global }} y_{C O P, \text { global }} 0\right]^{T}$ is the Cartesian position of the global COP in double support, $\mathbf{X}_{C O P, 0}=\left[\begin{array}{lll}x_{C O P, 0} & y_{C O P, 0} & 0\end{array}\right]^{T}$ and $\mathbf{X}_{C O P, 1}=\left[\begin{array}{lll}x_{C O P, 1} & y_{C O P, 1} & 0\end{array}\right]^{T}$ are the Cartesian positions of left and right foot COP respectively, $R_{n, 0}$ and $R_{n, 1}$ are the normal reaction forces under left and right foot respectively and $R_{n, 0}+R_{n, 1}$ represents the total body weight. Equation (1) shows that we can modify COP location via the COP location of each contact area, left and right, or via normal forces distribution between the support zones.

To prevent falling from higher perturbations, a third mechanism is identified : stepping [5][6] in order to have a new support polygon, new boundaries for COM to remain stable. Hof et al. [7] give a formulation of the equations of motion for balance in a very general form based on inverted pendulum model. These mechanisms, in particular the third one, are very efficient in sagittal plan but in frontal plan, they are limited. For instance, if we are highly pushed on the side, all the weight is transferred on the opposite leg, therefore we cannot move this leg to enlarge the base of support. To recover balance we need to jump on the side. The jump part will not be handled by our balance control.

For safety, it is important that the machine does not hurt the operator by apply- 
ing efforts in the wrong way. We try to design the balance control in order to have strategies close to human's.

\subsection{Instantaneous capture point}

\subsubsection{Definition}

The condition of balance for static stability "the vertical projection of COM on the ground (GCOM) should be within the convex polygon shaped by the feet called support polygon" is valid only when dynamic effects can be neglected as velocity and acceleration are limited. A more important point called instantaneous capture point [8][9] or extrapolated center of mass [10] is required inside the support polygon to allow the system to come to a stop. To make it easier to understand, it represents for a linear inverted pendulum model, the position that the inverted pendulum base needs to move instantaneously to stop and for a humanoid, it is the place that the step should be taken to come to a stop. The Cartesian position of the instantaneous capture point (ICP) is defined as (Fig.1 (a)):

$$
\mathbf{X}_{I C P}=\mathbf{X}_{G C O M}+\frac{\dot{\mathbf{X}}_{G C O M}}{\omega_{0}}
$$

with $\omega_{0}=\sqrt{\frac{g}{z_{C O M}}}$ where $\mathbf{X}_{G C O M}=\left[x_{C O M} y_{C O M} 0\right]^{T}$ is the Cartesian position of the vertical projection of COM, $\dot{\mathbf{X}}_{G C O M}=\left[\begin{array}{ll}\dot{x}_{C O M} & \dot{y}_{C O M} 0\end{array}\right]^{T}$ is the Cartesian velocity of GCOM, $g$ is the gravitational acceleration and $z_{\mathrm{COM}}$ is the height of COM.

\subsubsection{Dynamics}

To study human balance or control bipedal walking robots, the linear inverted pendulum (LIP) model from Kajita is widely used [11]. This model uses different assumptions such as :

- the system is modeled as a point mass corresponding to COM

- COM is kept at a constant height with a massless telescoping leg

- the base joint of the pendulum COP is torque free

For the horizontal acceleration of the COM, the equations of force equilibrium of LIP model give :

$$
\ddot{\mathbf{X}}_{G C O M}=\omega_{0}^{2}\left(\mathbf{X}_{G C O M}-\mathbf{X}_{C O P}\right)
$$

By differentiating the definition of ICP and manipulating the equations, it leads to these two important equations [12][13][9][14] : 


$$
\dot{\mathbf{X}}_{G C O M}=-\omega_{0}\left(\mathbf{X}_{G C O M}-\mathbf{X}_{I C P}\right)
$$

and

$$
\dot{\mathbf{X}}_{I C P}=\omega_{0}\left(\mathbf{X}_{I C P}-\mathbf{X}_{C O P}\right)
$$

(4) shows that GCOM has a stable first-order open loop dynamics : GCOM converges toward ICP and (5) shows that ICP has an unstable first-order open loop dynamics, it illustrates ICP revulsion regarding to COP. Via its ICP, we are able to control GCOM movements and consequently, the machine's motion : we calculate a COP that creates the desired movement, this implies that ICP moves on a known straight line and GCOM simply follows.

In this paper, we consider a fully actuated exoskeleton standing on a flat ground without considering the whole coupled system \{operator+exoskeleton\} and we suppose the exoskeleton transparent, it means that it can be driven easily by an operator. In order to preserve balance, thus the safety of the operator, we will only focus on how the exoskeleton should react to perturbations, which can be from the operator. We present a balance control for an exoskeleton assisting well and able persons so we need to preserve their comfort to the maximum. Control ICP allows us to take advantage of the natural dynamics of LIP model and therefore to have a smooth control of system's COM. From now, we will only consider ICP instead of COM since we know that COM naturally converges toward it.

(a)

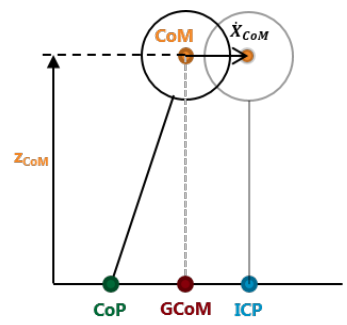

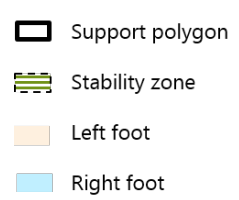

(b)

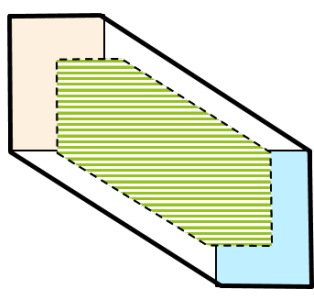

Fig. 1 (a) LIP model : Definition of ICP. (b) Stability zone.

\section{BALANCE CONTROL}

\subsection{Strategies and detection of a loss of balance situation}

If ICP exceeds the boundaries of the support polygon (SP), stability can no longer be maintained without stepping. In order to preserve the stability to the maximum, we do not let ICP to move close to SP boundaries except if the operator forces the exit. We define an area called stability zone (SZ) where no balance control is neces- 
sary, because ICP is far enough from limits to remain in a stable situation. We chose this zone such as it represents $70 \%$ of the support polygon (Fig.1 (b)).

To detect a loss of balance situation, we track ICP position and we consider, three different cases (Fig.2) :

- ICP is inside SZ : the system is stable, balance control is disabled.

- ICP is outside SZ but inside SP : the system is still stable but gets closer to an unstable situation, the balance control brings back ICP inside SZ.

- ICP is outside SP : the system is unstable, the system is going to fall if nothing is done. Human's reflex is to step in order to prevent from falling. We design the balance control to help him to balance and to reduce efforts he should produce to move.

In accordance with ICP position, the balance control will adapt to the situation.

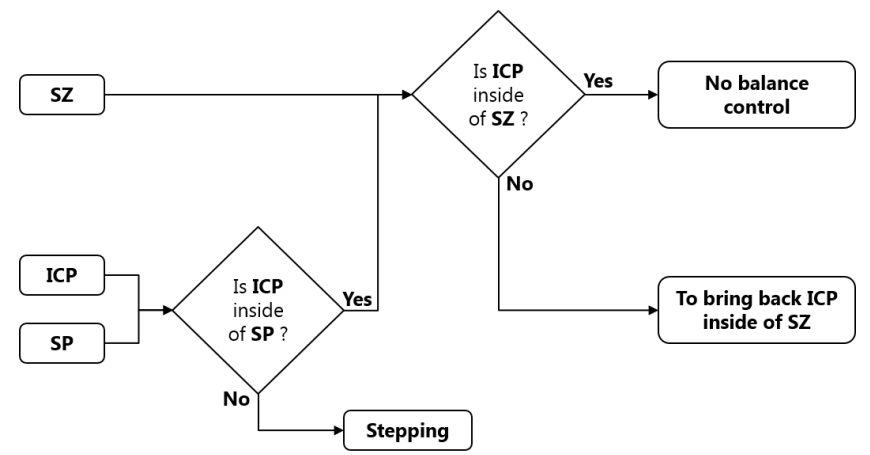

Fig. 2 Balance control logic diagram. (ICP = Instantaneous Capture Point, SP = Support Polygon, $\mathrm{SZ}=$ Stable Zone)

\subsection{Balance control for a load carrying exoskeleton}

To design a balance control for this kind of device that ensure human's movements freedom to the maximum in regard of the machine's transparency and limitations, we need to take into consideration operator's intentions. From the machine's point of view, the operator behaves like an external force applied at attached points that accelerates the exoskeleton's COM and implicitly moves its ICP and we try to assist the recovery of balance of the system. Consequently, we will only focus on how to control stance leg(s) because swing leg is supposed to be driven by the operator as the system is transparent. 
We suppose that the operator is well fastened to the exoskeleton so that he orders the velocity of the machine and we have $\dot{\mathbf{X}}_{G C O M, e}=\dot{\mathbf{X}}_{G C O M, o p}$, we can write :

$$
\mathbf{X}_{I C P, e}=\mathbf{X}_{G C O M, e}+\frac{\dot{\mathbf{X}}_{G C O M, o p}}{\omega_{0, e}}
$$

with $\omega_{0, e}=\sqrt{\frac{g}{z_{C O M, e}}}$ where the subscripts $e_{e}$ and ${ }_{o p}$ mean 'exoskeleton' and 'operator' respectively.

\section{CAPTURE POINT PLACEMENT}

One of the important part of the control is to regulate ICP location and keep it in a stable situation. Depending on the situation, we first determine a desired position for the ICP, then thanks to a simple first order control law and equation (4), a desired $\mathrm{COP}$ is calculated. The goal is to calculate the correction wrench that the actuators should produce to send the real COP to the desired one and therefore, to create the desired motion.

\subsection{Desired Instantaneous Capture Point}

The correction motion depends on the location of the desired ICP. The balance control idea is to keep ICP in SZ either by creating a correction motion that brings it back into SZ or by modifying the support polygon with a step to make it safe. The different cases are :

1. ICP is inside of $\mathbf{S P}$ and inside of $\mathbf{S Z}$ : There is no correction, $\mathbf{X}_{I C P}^{(d)}=\mathbf{X}_{I C P}$ where the superscript ${ }^{(d)}$ means 'desired'.

2. ICP is inside of SP and outside of $\mathbf{S Z}: \mathbf{X}_{I C P}^{(d)}$ is the projection of the current ICP on SZ based on a Euclidean heuristic.

3. ICP is outside of SP : We choose to trigger a step because we know the system is unstable and the single support phase can begin. To determine $\mathbf{X}_{I C P}^{(d)}$, we extrapolate the location of ICP. We integrate the equation 5 and given the current location of ICP, we can deduce the direction of the motion at time $t_{0}$ when ICP go out of SP for the first time. We calculate the location of ICP at $t_{s}$, minimal time to make a step, if nothing is done to correct or modify its trajectory :

$$
\mathbf{X}_{I C P}\left(t=t_{s}\right)=\left(\mathbf{X}_{I C P}\left(t=t_{0}\right)-\mathbf{X}_{C O P, 0}\right) e^{\omega_{0} t_{s}}+\mathbf{X}_{C O P, 0}
$$

To maximize the stability margin at the end of single support phase, when the swing foot reaches the ground, ICP should be at the center of the support poly- 
gon, so $\mathbf{X}_{I C P}^{(d)}=\mathbf{X}_{I C P}\left(t=t_{s}\right)=$ center of the next double support polygon. Therefore, we can determine the ideal location of the step $\mathbf{X}_{\text {step }}$ and then, the ideal next double support polygon at $t=t_{S}$ (Fig.4). As long as the swing foot does not reach the ground and the single support is not finished, $t_{0}$, and so $\mathbf{X}_{\text {step }}$, remains the same : $t_{0} \leq t \leq t_{\text {end }}$. Note that the step has not to be made at $t=t_{s}$ : the desired ICP indicates the direction of motion of the system. Our goal is to encourage the motion in a desired direction, not to force a movement.

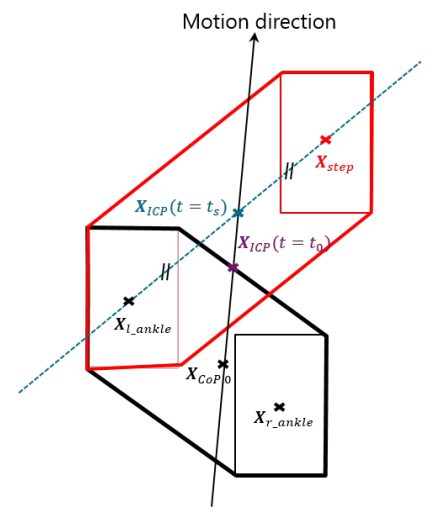

Fig. 3 Determination of the ideal next double support polygon.

\subsection{Desired Center of Pressure of LIP Model}

Our objective is now to deduce articular torques that the legs should produce to reach the desired ICP. Torques will place COP at a specific location which will modify ICP velocity. We can write ICP velocity as :

$$
\dot{\mathbf{X}}_{I C P}=K_{I C P} \cdot\left(\mathbf{X}_{I C P}^{(d)}-\mathbf{X}_{I C P}\right)
$$

where $K_{I C P}$ is the proportional gain.

Basing on LIP model, we deduce COP position by equalizing equations (5) from equations of motion of LIP and (8) :

$$
\omega_{0}\left(\mathbf{X}_{I C P}-\mathbf{X}_{C O P}\right)=K_{I C P} \cdot\left(\mathbf{X}_{I C P}^{(d)}-\mathbf{X}_{I C P}\right)
$$




$$
\mathbf{X}_{C O P}=\mathbf{X}_{I C P}-\frac{K_{I C P}}{\omega_{0}} \cdot\left(\mathbf{X}_{I C P}^{(d)}-\mathbf{X}_{I C P}\right)
$$

with $K_{I C P}>0$ in order to preserve the direction of the motion, else $K_{I C P}$ is not consistent with system dynamic. COP must be inside SP in other case, the stance feet will rotate. Therefore, COP can't physically be outside SP, if $\mathbf{X}_{C O P}$ is outside, we use a heuristic to project it on SP.

\subsection{Computation of Correction Wrench}

This section deals with how to compute the correction wrench that allows us to reach our ICP objective. In the previous section, we determined the location of the $\mathrm{COP}$ that moves ICP to the desired ICP. It means that placing the current COP there creates an acceleration at $\mathrm{COM}$ as following (eq.3) :

$$
\ddot{\mathbf{X}}_{G C O M}=\omega_{0}^{2}\left(\mathbf{X}_{G C O M}-\mathbf{X}_{C O P}\right)
$$

So we can easily write the correction wrench that brings the system to a stable situation as :

$$
\mathbf{W}_{c o r r, G}=\left[\begin{array}{c}
\mathbf{F}_{c o r r} \\
\mathbf{M}_{c o r r}, G
\end{array}\right]=\left[\begin{array}{c}
m \ddot{\mathbf{X}}_{G C O M} \\
\mathbf{0}
\end{array}\right]
$$

with

$$
\mathbf{F}_{\text {corr }}=m \ddot{\mathbf{X}}_{G C O M}=\frac{m g}{z_{C O M}}\left(\mathbf{X}_{G C O M}-\mathbf{X}_{C O P}\right)
$$

where $m$ is the mass of the system and $\mathbf{X}_{C O P}$ is the one calculated previously. Note that the generated correction wrench is an horizontal force applied on COM and have zero moment around it.

So we can write the dynamic equation :

$$
\mathbf{A d}_{G} \mathbf{W}_{\text {ground }, P}+\mathbf{W}_{\text {weight }, G}-\mathbf{W}_{\text {corr }, G}=\mathbf{W}_{\text {dyn }, G}
$$

where

- $\mathbf{A d}_{G}$ : adjoint transformation associated with COP to COM.

- $\mathbf{W}_{\text {ground,P }}$ : wrench of ground reaction force applied at COP.

- $\mathbf{W}_{\text {weight }, G}$ : wrench of gravity force applied at COM where $m$ is the mass of the system.

- $\mathbf{W}_{c o r r, G}$ : correction wrench applied at COM which we determined.

- $\mathbf{W}_{d y n, G}:$ dynamic wrench applied at COM.

Now $\mathbf{W}_{c o r r, G}$ is known, we obtain the entire effort that the actuators need to produce. Let's :

$$
\mathbf{W}_{\text {act }}=\mathbf{W}_{\text {corr }}-\left(\mathbf{W}_{\text {weight }, G}+\mathbf{W}_{\text {dyn }, G}\right)
$$


$\mathbf{W}_{\text {corr }}$ is produced to help the operator and $\left(\mathbf{W}_{\text {weight }, G}+\mathbf{W}_{\text {dyn }, G}\right)$ is necessary for the machine to care its weight and an additional load which is its first job before assisting.

\section{COEFFICIENT OF LEG CONTRIBUTION}

Once $\mathbf{W}_{a c t}$ is known and to calculate joint torques, we adopt the intuitive strategy used by Pratt et al. [15] of distributing the forces between the two legs. The leg support fractions are two coefficients $\left(\alpha_{0} ; \alpha_{1}\right)(0=$ left, $1=$ right $)$ describing the fraction of the desired wrench to be exerted by each leg with $\alpha_{0}+\alpha_{1}=1$. $\alpha_{i}=0$ means that leg $i$ is the swing one. In [16] the forces are distributed according to the following geometric ratio :

$$
\alpha_{i}=\frac{\left\|\mathbf{X}_{G C O M}-\mathbf{X}_{1-i}\right\|_{2}}{\left\|\mathbf{X}_{0}-\mathbf{X}_{1}\right\|_{2}}
$$

with $i \in\{0,1\}$ and where $\mathbf{X}_{0}$ and $\mathbf{X}_{1}$ are respectively the center of left and right foot polygon. Expression (16) shows that if the mass gets closer to a foot, the related leg contribution increases to support it. In [14], Pratt et al. use the ratio of COPs distances but it supposed to know or estimate left and right COP position.

However these coefficients do not take into consideration perturbations. We propose a coefficient that takes into account the direction of perturbations using ICP, and the limits of SP to transfer the load from a leg to the other appropriately when needed.

\subsection{Description of the situation}

Giving a feet configuration, we will resist to a perturbation by supporting more efforts on one leg than the other : if we are pushed on the right side, the contribution of the right leg will be higher than the one on the left. We introduce a first coefficient $\beta$ which represents the proximity to a foot :

$$
\beta_{i}=\frac{\left\|\mathbf{X}_{I C P}^{(p)}-\mathbf{X}_{1-i}\right\|_{2}}{\left\|\mathbf{X}_{0}-\mathbf{X}_{1}\right\|_{2}}
$$

with $i \in\{0,1\}$ and where $\mathbf{X}_{I C P}^{(p)}$ is the projection of ICP on feet axe.

$\beta$ indicates which leg should produce more efforts to resist the perturbation. However, taking into account the feet positions only is not enough : ICP can get closer to the support polygon's boundaries and still have a $\beta \simeq 0.5$. Indeed, when ICP leaves the polygon, a step is taken. To anticipate this action, we need to transfer the weight to the future support leg to allow swing foot take-off. Therefore, we introduce a 
second coefficient $\gamma$ as following :

$$
\gamma=\frac{\left\|\mathbf{X}_{I C P}^{(p)}-\mathbf{X}_{I C P}\right\|_{2}}{\left\|\mathbf{X}_{I C P}^{(p)}-\mathbf{X}_{\text {bound }}\right\|_{2}}
$$

where $\mathbf{X}_{\text {bound }}$ represents the projection of ICP on the closer boundary. This way, we have all necessary information to describe the location of ICP in SP and determine the coefficients of leg contribution $\alpha$.

(a)

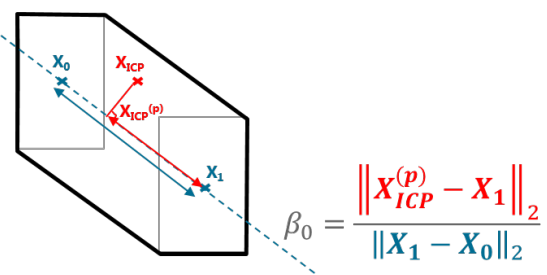

(b)

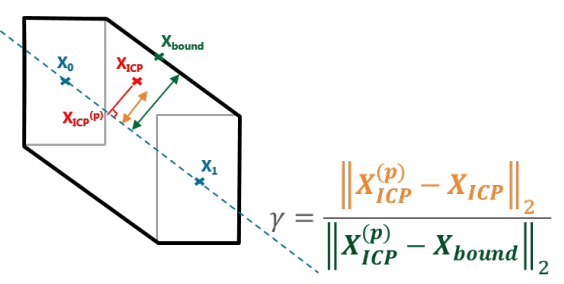

Fig. 4 (a) Definition of $\beta$. (b) Definition of $\gamma$.

\subsection{Stabilization}

We build $\alpha$ in function of $\beta$ and $\gamma$. Let's consider ICP on feet axe : we want $\alpha_{0}=\beta_{0}$. However, if ICP moves away from the axe to join SP limits, $\alpha$ has to converge to 1 for the closest leg :

$$
\alpha_{0}(\gamma)=\left\{\begin{array}{cl}
1-\exp \left(\lambda\left(\gamma+\gamma_{\beta_{0}}\right)\right) & \text { if }\left\|\mathbf{X}_{I C P}-\mathbf{X}_{0}\right\|_{2} \leq\left\|\mathbf{X}_{I C P}-\mathbf{X}_{1}\right\|_{2} \\
\exp \left(\lambda\left(\gamma+\gamma_{\beta_{0}}\right)\right) & \text { if } \left.\left\|\mathbf{X}_{I C P}-\mathbf{X}_{0}\right\|_{2}>\left\|\mathbf{X}_{I C P}-\mathbf{X}_{1}\right\|_{2}\right)
\end{array}\right.
$$

where $\lambda$ is the convergence velocity and $\gamma_{\beta_{0}}$ is the necessary offset to have $\alpha_{0}(\gamma=$ $0)=\beta_{0}$. With this $\alpha$, the weight transfer is handled and the leg is ready to anticipate the perturbation and stabilize the system.

At this point, we have the contribution of each leg to split the efforts in the following way :

$$
\mathbf{W}_{a c t, i}=\alpha_{i} \cdot \mathbf{W}_{a c t}
$$

Then we use the Jacobian matrix to calculate joint torques to apply to the machine. 


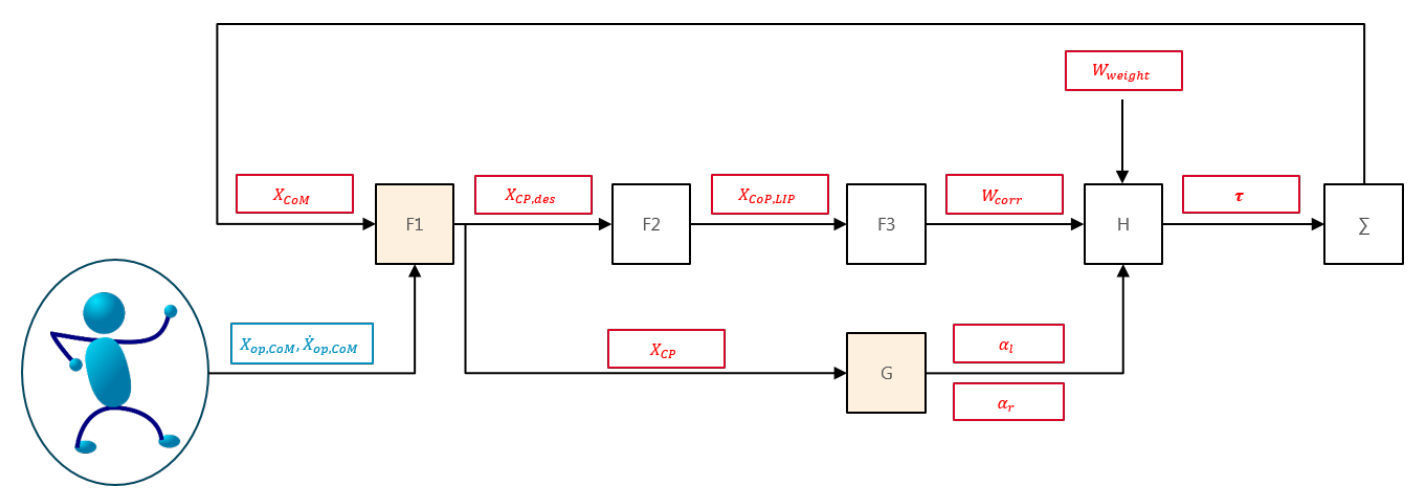

Fig. 5 Open-loop balance control scheme.

\section{SIMULATIONS}

We consider a fully-actuated exoskeleton of twelve degrees of freedom with six per leg : hip flexion/extension and abduction/adduction, thigh rotation, knee flexion/extension, ankle flexion/extension and prono-supination. Since we can't really simulate an autonomous operator, to overcome his presence in the simulator, we use a Cartesian external force $\mathbf{F}_{\text {push }}$ applied at the center of the phantom during $t_{\text {push }}$ seconds to represent when he pulls the machine.

We test different situations with following parameters $t_{s}=1 \mathrm{~s}, K_{I C P}=100$ (x points to the right, $\mathrm{y}$ forward and $\mathrm{z}$ up) with the feet configuration in figure 6 :

1. The operator moves forwards : ICP inside SZ, the balance control does not need

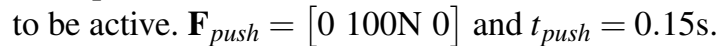

2. He moves forwards and diagonally : ICP leaves SZ, the balance control brings ICP back into SZ. $\mathbf{F}_{\text {push }}=[100100 \mathrm{~N} 0]$ and $t_{\text {push }}=0.15 \mathrm{~s}$.

3. He moves faster forwards : he forced ICP to leave SZ and then SP, the balance control detects that a step will be taken to recover balance (left leg support). $\mathbf{F}_{\text {push }}=\left[\begin{array}{lll}0 & 350 \mathrm{~N} & 0\end{array}\right]$ and $t_{\text {push }}=0.075 \mathrm{~s}$.

In the simulation, at $t=0$ s we unlock exoskeleton's joints and the balance control is enabled and it converges toward an stable state : ICP inside of SZ and $\left(\alpha_{0} ; \alpha_{1}\right) \approx$ $(0.49 ; 0.51)$. On the graphs, dotted lines represents the beginning of each phase previously mentionned :

- Fig.7 (a) and (b) highlight the perturbations shown on COM velocity graphs and the consequences on desired and current ICP trajectories. The most interesting is ICP trajectories : current ICP well tracks on desired ICP. At the first stage, ICP is inside SZ, therefore $\mathbf{X}_{I C P}^{(d)}=\mathbf{X}_{I C P}$. The second perturbation leads ICP to get out of SZ: the balance control brings it back in order to guarantee the stability. At the third stage, ICP exits SZ then SP. A step is taken. Current ICP converges toward 

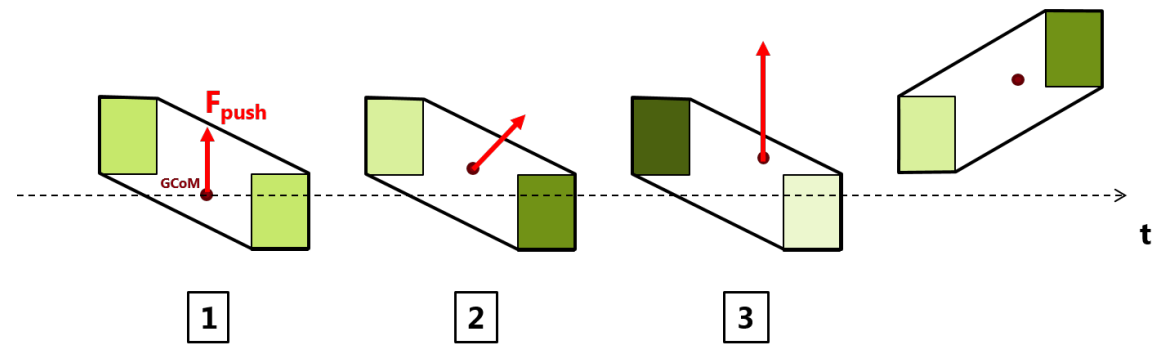

Fig. 6 Proceedings of the simulation scheme, perturbations are accumulated. The more the foot polygon is dark green, the more the leg contribution coefficient is close to 1 .

(a)
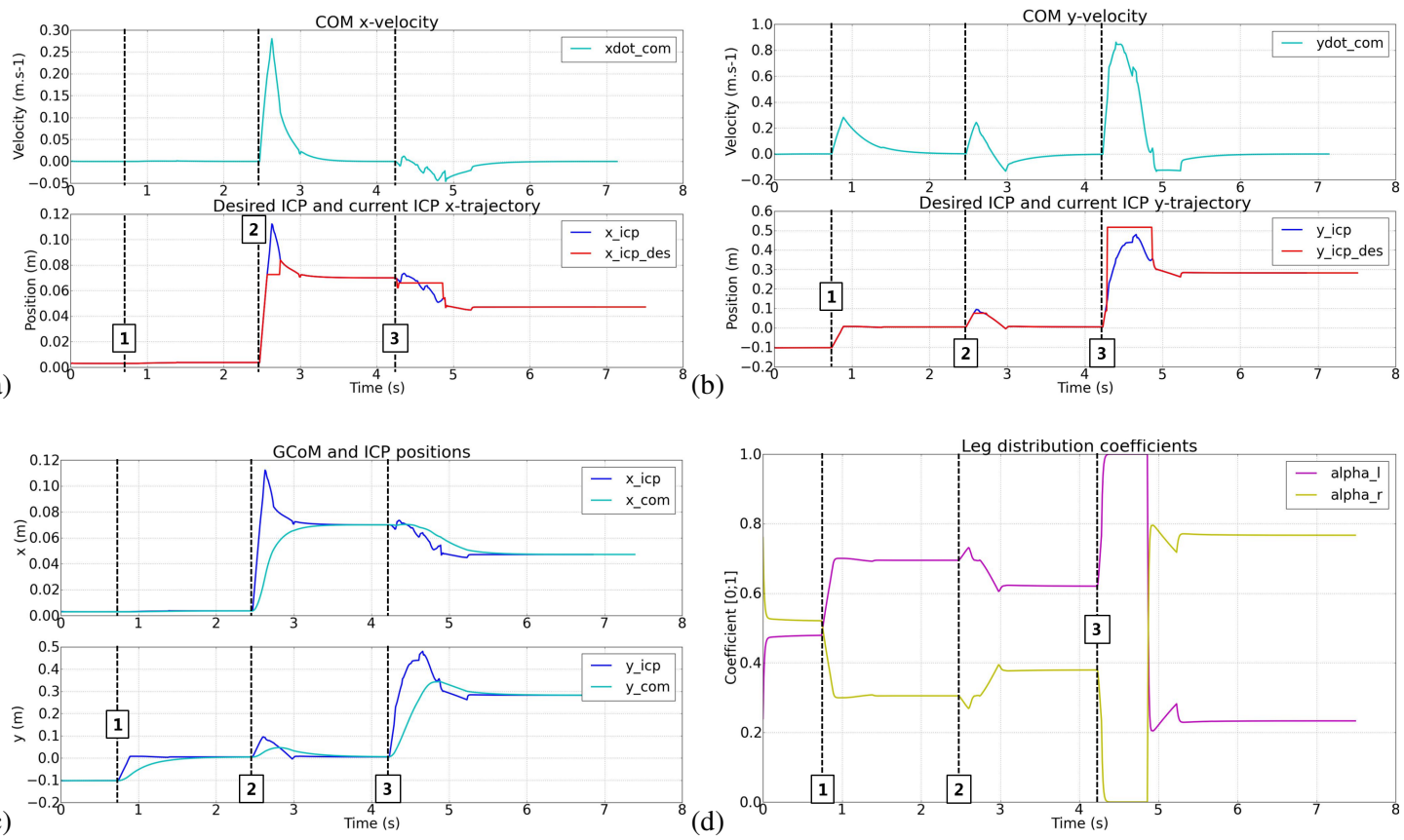

Fig. 7 (a) and (b) Desired and current ICP position in function of the perturbations on COM represented by COM velocity. (c) Trajectory of COM and ICP. (d) Coefficient of leg distribution.

desired ICP but we don't wait for its complete convergence to switch from single support phase to double support phase.

- Fig.7 (c) represents the pursuit of COM for ICP in a natural convergence. It shows that our balance control regulates indirectly and smoothly COM trajectory in order to control the movement according to a direction.

- Fig.7 (d) shows the evolution of leg contribution coefficients $\left(\alpha_{0} ; \alpha_{1}\right)$. Magenta line represents left leg and yellow line, right leg. The first perturbation is forward : ICP get close to left foot, $\alpha_{0}$ increases. The second one is forward and to the right : $\alpha_{1}$ increases. Then, at the third stage, the system is pushed forward so ICP 
is close to left foot and reaches SP limits. All the weight is transferred on left leg, in order to trigger the swing phase.

\section{CONCLUSION}

We presented a balance control based on the control of the instantaneous capture point of the exoskeleton. It allows the machine to detect a loss of balance situation and to imitate human mechanisms in order to help him to recover his balance. In this paper, we supposed a fully-actuated exoskeleton and we neglected the interaction with the operator. Undergoing works deal with underactuaction (lightening machines implies reducing the number of actuators) or overactuaction which leads to opposite efforts produced by both legs. Moreover, we need experiments to validate that our balance control is well accepted by the operator.

\section{References}

1. A. M. Dollar and H. Herr, Lower extremity exoskeletons and active orthoses : challenges and state-of-the-art, IEEE Transactions on robotics, vol. 24, (2008)

2. R. Bogue, Exoskeletons and robotic prosthetics : a review of recent developments, Industrial Robot : an international journal, 421-427, (2009)

3. P. Garrec, Y. Perrot, D. Ponsort, F. Coste, S. Grygorowicz, A. Riglet, Lower exoskeleton, Patent WO 2013057057, (2013)

4. B. Moriniere, A. Verney, N. Abroug, P. Garrec and Y. Perrot, EMY: a dual arm exoskeleton dedicated to the evaluation of Brain Machine Interface in clinical trials, IEEE/RSJ International Conference on Intelligent Robots and Systems (IROS), pp. 5333-5338, (2015)

5. D. A. Winter, Human balance and posture control during standing and walking, Gait \& Posture, vol. 3, 193-214, (1995)

6. F. B. Horak and L. M. Nashner, Central programming of postural movements : adaptation to altered support surface configurations, Journal of Neurophysiol, vol. 55, no. 6, 1369-1381, (1986)

7. A. L. Hof, The equations of motion for a standing human reveal three mechanisms for balance, Journal of biomechanics, vol. 40, 451-457, (2005)

8. J. Pratt, J. Carff, S. Drakunov and A. Goswami, Capture point : a step toward humanoid push recovery, In Proc. IEEE International Conference on humanoid robotics, 200-207, (2006)

9. T. Koolen, T. de Boer, J. Rebula, A. Goswami and J. Pratt, Capturability-based analysis and control of legged locomotion, Part 1 : Theory and application to three simple gait models, The international journal of robotics research, (2012)

10. A. L. Hof, M. G. J. Gazendam and W. E. Sinke, The condition for dynamic stability, Journal of biomechanics, vol. 38, 1-8, (2004)

11. S. Kajita, F. Kanehiro, K. Kaneko, K. Fujiwara, K. Harada, K.Yokoi and H. Hirukawa, In Proc. IEEE International Conference on robots and automation, (2003)

12. A. L. Hof, The 'extrapoled center of mass' concept suggests a simple control of balance in walking, Human movement science, vol. 27, 112-125, (2007)

13. J. Englsberger, C. Ott, M. A. Roa, A. Albu-Schffer and G. Hirzinger, Bipedal walking control based on capture point dynamics, In Proc. IEEE International Conference on intelligent robots and systems, (2011) 
14. J. Pratt, T. Koolen, T. de Boer, J. Rebula, S. Cotton, J. Carff, M. Johnson and P. Neuhaus, Capturability-based analysis and control of legged locomotion, Part 2: Application to M2V2, a lower-body humanoid, The international journal of robotics research, (2012)

15. J. Pratt and G. Pratt, Intuitive control of planar bipedal walking robot, In Proc. IEEE International Conference on Robotics and Automation, (1998)

16. J. L. Racine, Control of a lower limb extremity exoskeleton for human performance amplification, PhD dissertation, Univ. of California, Berkeley (2003) 